\title{
Attitudes and perceptions of the general Malaysian public regarding family presence during resuscitation
}

\author{
Keng Sheng $\underline{\text { Chew }}{ }^{1}$, MD, MMed, Zuhailah Abdul $\underline{G h a n i}^{1}$, MD, MMed
}

INTRODUCTION Family presence (FP) during resuscitation is an increasingly favoured trend, as it affords many benefits to the critically ill patient's family members. However, a previously conducted study showed that only $15.8 \%$ of surveyed Malaysian healthcare staff supported FP during resuscitation.

METHODS This cross-sectional study used a bilingual self-administered questionnaire to examine the attitudes and perceptions of the general Malaysian public toward the presence of family members during resuscitation of their loved ones. The questionnaires were randomly distributed to Malaysians in three different states and in the federal territory of Kuala Lumpur.

RESULTS Out of a total of 190 survey forms distributed, 184 responses were included for analysis. Of the 184 respondents, $140(76.1 \%)$ indicated that they favoured FP during resuscitation. The most common reason cited was that FP during resuscitation provides family members with the assurance that everything possible had been done for their loved ones ( $n=157,85.3 \%)$. Respondents who had terminal illnesses were more likely to favour FP during resuscitation than those who did not, and this was statistically significant $(95.0 \%$ vs. $73.8 \% ; p=0.04)$.

CONCLUSION FP during resuscitation was favoured by a higher percentage of the general Malaysian public as compared to Malaysian healthcare staff. This could be due to differences in concerns regarding the resuscitation process between members of the public and healthcare staff.

Keywords: family presence, invasive procedures, Malaysian, resuscitation

\section{INTRODUCTION}

Family presence (FP) is described as "the presence of family in a patient care location that affords visual or physical contact with the patient during invasive procedures or resuscitation events". ${ }^{(1)}$ FP during resuscitation is an increasingly favoured trend among patients and their family members due to the benefits it affords. For instance, it allows the patient's family members to comfort their loved ones during invasive procedures and the resuscitation process. It also provides space for the grieving process and an opportunity for the patient's family members to bid farewell should the patient be on the verge of dying. ${ }^{(1,2)}$ In addition to the aforementioned benefits, FP can help increase the family members' understanding of the seriousness and reality of the patient's medical condition, thus improving the quality of the decisions made. ${ }^{(3)} \mathrm{A}$ Turkish study conducted on 420 families found that up to $66.4 \%$ of family members would like to be present during resuscitation of their loved ones. ${ }^{(4)}$ However, a study conducted on the general public's perception on witnessed cardiopulmonary resuscitation suggests that the opinion of the general public on this issue is more divided than that of patients' family members. ${ }^{(2)}$ Among healthcare staff, nurses were found to be more in favour of FP during resuscitation than physicians. ${ }^{(5)}$

One of the authors of the present study was involved in a survey conducted to evaluate the attitude of Malaysian healthcare staff toward FP during resuscitation. ${ }^{(6)}$ In that study, only $15.8 \%$ of the 270 healthcare staff who participated in the survey showed a positive attitude toward FP during resuscitation, although
$38.5 \%$ of them agreed that the patient's family members have a right to be present during resuscitation and procedures. The study also revealed that doctors were more than twice as likely as paramedics to agree with the practice of FP. This is likely due to the work culture in Malaysian healthcare systems, where paramedics usually adopt a 'follow-the-leader' attitude in their work practices. ${ }^{(6)}$ As the perception of Malaysian healthcare staff towards FP during resuscitation had been studied, we conducted a cross-sectional study to determine the attitude of the adult (aged $\geq 18$ years) Malaysian population regarding the same issue. We also sought to determine the factors affecting their decisions.

\section{METHODS}

A bilingual (English and Malay language) self-administered questionnaire was used for the purpose of this study. The questionnaire was developed based on the questionnaire used in a previous study. ${ }^{(6)}$ To assess the reliability and validity of the questionnaire, a pilot study that involved 60 participants was conducted in July 2011 with the help of biostatisticians in our medical school. After the reliability and validity of the questionnaires were found to be satisfactory, the questionnaires were then randomly distributed to Malaysians in three different states of Malaysia (i.e. Melaka, Penang and Kelantan), as well as the federal territory of Kuala Lumpur. Participants were members of the general Malaysian public who were randomly approached in different settings, such as the waiting area of clinics in Kuala Lumpur General Hospital, school premises, government offices, and the neighbourhood of certain pre-identified housing areas

${ }^{1}$ Emergency Medicine Department, School of Medical Sciences, Universiti Sains Malaysia, Malaysia

Correspondence: Dr Chew Keng Sheng, Senior Lecturer, c/o Emergency Medicine Department, School of Medical Sciences, Universiti Sains Malaysia, 16150 Kubang Kerian, Malaysia.cksheng74@yahoo.com 
in Kuala Lumpur and the three states. Participants were selected through convenience sampling.

The purpose of the survey was explained verbally, and consent was obtained from every person who volunteered to participate in the study. The survey forms were handed out in person by one of the authors of the study. After the form was completed by the participants, they were sealed in an opaque envelope and returned. Survey forms that were not filled (i.e. blank), illegible or damaged were excluded from the data analysis. The study was conducted for a period of six months, from June 2012 to December 2012. Approval for the study was obtained from the research ethics committee board of our institution. Statistical analyses were conducted using the Statistical Package for the Social Studies version 16.0 (SPSS Inc, Chicago, IL, USA).

\section{RESULTS}

Of the 190 survey forms distributed, 184 were used for data analysis (six forms were excluded, as they were either blank, illegible or damaged). The mean age of the participants was 31.6 (range 19-55) years, and a larger proportion was female $(n=116$, $63.0 \%)$. In terms of the participants' educational level, $4(2.2 \%)$ had a master's or PhD degree, 38 (20.7\%) had basic degrees, and 85 (46.2\%) had diplomas. The remaining 57 (31.0\%) participants had secondary school level education.

A total of $140(76.1 \%)$ participants answered 'yes' to the question of whether family members should be allowed to be present during resuscitation, and 150 (81.5\%) answered 'yes' to the question of whether family members have a right to be present during resuscitation (Table I). With regard to the benefits of allowing FP during resuscitation, the most common reason given was that it provides family members with the assurance that everything possible has been done for their loved ones ( $n=157,85.3 \%)$. This is followed by the advantage of allowing the final religious rites to be performed for the patients $(n=99$, $53.8 \%$ ). Only one participant responded that FP does not offer any benefit. Participants who had terminal illnesses were more likely to favour FP during resuscitation than those who did not, and this difference was statistically significant $(95.0 \%$ vs. $73.8 \%$, $\mathrm{p}=0.04$; Table II).

\section{DISCUSSION}

Although FP during resuscitation has already been advocated in the West, ${ }^{(7)}$ the concept is still relatively new in Asia. ${ }^{(8)}$ However, as the general public becomes more knowledgeable concerning diseases because of the rapid dissemination of information via information technology, the traditional approach of excluding patients and family members from medical decision-making is increasingly out of fashion, even in the Asian setting. Patients and family members are also less likely to accept verdicts and decisions from healthcare authorities without question.

In the present study, we found that a majority of the Malaysians surveyed ( $\mathrm{n}=140,76.1 \%$ ) believed that family members should be allowed to witness resuscitation and invasive procedures. This is in contrast to the findings of a previous study conducted on Malaysian healthcare staff, in which only $15.8 \%$
Table I. Descriptive analysis of the attitudes of the general Malaysian public $(n=184)$ toward family presence during resuscitation.

Question
Should the presence of family members be
allowed during resuscitation?
Yes
No
Do family members have a right to be present
during resuscitation?
Yes
No
In the event of resuscitation, when should fam
members be allowed to be present?
Never
After all necessary invasive procedures have
performed
During the entire resuscitation process
Which of the following procedures should the
family members be allowed to witness?
Bloody procedures
Non-bloody procedures
Cardiopulmonary resuscitation
Examination of private parts
None

No. (\%)

Do family members have a right to be present

during resuscitation?

Yes

$150(81.5)$

$34(18.5)$

In the event of resuscitation, when should family members be allowed to be present?

After all necessary invasive procedures have been

$104(56.5)$

What is the ideal number of family members that should be allowed to witness the resuscitation?

0

1

$2-3$

$>3$

Should staff provide emotional and psychological support for the family members that are present? Yes

$21(11.4)$

$64(34.8)$

$85(46.2)$

$14(7.6)$

No

$174(94.6)$

$10(5.4)$

In your opinion, which of the following are benefits of allowing family presence?

It helps to assure family members that everything possible has been done

It aids in the family members' grieving process

It promotes mutual understanding between family members and the healthcare staff

It enables family members to give final religious

rites

None

$157(85.3)$

25 (13.6)

$20(10.9)$

$99(53.8)$

$1(0.5)$

In your opinion, which of the following are reasons

to not allow family presence?

It is a traumatic experience

It may have medicolegal implications

$129(70.1)$

$60(32.6)$

$30(16.3)$

$73(39.7)$

$15(8.2)$

None

Which of the following family members should be allowed in the resuscitation room?

Parents of paediatric patients

Siblings of paediatric patients

$174(94.6)$

$43(23.4)$

$155(84.2)$

125 (67.9)

Adult children of geriatric patients

Which of the following groups of patients

will benefit from family presence during

resuscitation?

Patients with acute illnesses

89 (48.4)

Patients with chronic illnesses

$135(73.4)$

Trauma patients

117 (63.6)

Terminally ill patients 
Table II. Univariate analysis of the association between various factors and the attitudes of the general Malaysian public toward family presence during resuscitation.

\begin{tabular}{|c|c|c|c|}
\hline \multirow[t]{2}{*}{ Factor } & \multicolumn{2}{|c|}{$\begin{array}{c}\text { Family presence } \\
\text { should be allowed* }\end{array}$} & \multirow[t]{2}{*}{ p-value ${ }^{+}$} \\
\hline & Yes & No & \\
\hline \multicolumn{4}{|l|}{ Gender } \\
\hline Male $(n=68)$ & $52(76.5)$ & $16(23.5)$ & 0.93 \\
\hline Female $(n=116)$ & $88(75.9)$ & $28(24.1)$ & \\
\hline \multicolumn{4}{|l|}{ Education level } \\
\hline Tertiary level $(n=127)$ & $96(75.6)$ & $31(24.4)$ & 0.81 \\
\hline $\begin{array}{l}\text { Secondary level and } \\
\text { below }(n=57)\end{array}$ & $44(77.2)$ & $13(22.8)$ & \\
\hline \multicolumn{4}{|l|}{ Participants who have } \\
\hline Yes $(n=20)$ & $121(73.8)$ & $43(26.2)$ & \\
\hline No $(n=164)$ & & & \\
\hline \multicolumn{4}{|c|}{$\begin{array}{l}\text { Participants with a family } \\
\text { member who has terminal } \\
\text { illnesses }\end{array}$} \\
\hline Yes $(n=35)$ & $28(80.0)$ & $7(20.0)$ & 0.55 \\
\hline No $(n=149)$ & $112(75.2)$ & $37(24.8)$ & \\
\hline \multicolumn{4}{|c|}{$\begin{array}{l}\text { Participants who have } \\
\text { previous experience of } \\
\text { witnessing a resuscitation } \\
\text { or invasive procedures }\end{array}$} \\
\hline Yes $(n=35)$ & $28(80.0)$ & $7(20.0)$ & 0.55 \\
\hline No $(n=149)$ & $112(75.2)$ & $37(24.8)$ & \\
\hline
\end{tabular}

*Data is presented as no. (\%). ${ }^{\dagger}$ All categorical univariate analysis were computed using chi-square test except for the category 'Participants with a family member who has terminal illness', for which Fisher's exact test was applied.

of the healthcare staff surveyed believed that family members should be allowed to witness resuscitation. ${ }^{(6)}$ This discrepancy is also conspicuous in the subcategory of those who agreed that family members should be allowed to be present throughout the entire resuscitation process. In the present study, 104 (56.5\%) participants from the general Malaysian public agreed that FP should be allowed in this scenario, whereas in the previous study involving Malaysian healthcare staff, only 3\% agreed to such a concession. ${ }^{(6)}$ A similar discrepancy was observed in another Asian study conducted in Singapore, in which Ong et al found that while $73.1 \%$ of the public preferred FP during resuscitation, only $10.6 \%$ of the surveyed healthcare staff were agreeable to such a concession. ${ }^{(8)}$

The reasons for such discrepancies remain elusive, although some postulations could be made based on an interesting qualitative study, conducted by sociologist Timmermans, on the perspectives of healthcare staff toward FP during resuscitation. ${ }^{(9)}$ Using in-depth interviews, Timmermans found that the perspectives of healthcare staff regarding resuscitation could be grouped into one of the following three categories - survival, bifurcated and holistic. Healthcare staff who subscribe to the survival perspective would generally view saving a human life to be the major objective of resuscitation. Those with the bifurcated perspective saw two separate goals for resuscitation - first, to save lives with all the technological means possible, and second, to take care of the needs of the patient's family. Healthcare staff who subscribe to the holistic perspective are equally concerned with several goals - survival of the patient, care of the patient's family and the need to keep family members informed. Although family members should be regarded as active participants in the resuscitation process, Timmermans believes that healthcare staff will not view the concept of FP during resuscitation favourably unless a paradigm shift takes place (from the survival perspective to the bifurcated or holistic perspective). Since the current perspectives of healthcare staff are diverse, it is suggested that FP during resuscitation be regarded as an option rather than a policy for the time being. ${ }^{(9)}$

In contrast to healthcare staff, family members are often concerned not only about the survival of the patient, especially if they are aware that the patient is terminally sick or has a low chance of survival, but also the opportunity to spend precious moments with their loved ones for the last time, bid them farewell and perform the final religious rites. The last concern is particularly important to Malaysian Muslims, as evidenced by the large number of Malaysian Muslims in the present study (53.8\%) who opined that this is an important reason for allowing FP during resuscitation. Therefore, it is probable that differences in expectation concerning the resuscitation process are reflected in the discrepancy of responses between family members and healthcare staff. However, one area that the majority of Malaysian members of the public and healthcare staff concur is that FP during resuscitation would reassure family members that everything possible has been done for their loved ones $(85.3 \%$ in the present study vs. $71.1 \%$ in Sheng et al's study $\left.{ }^{(6)}\right)$.

In the present study, we also found that participants with terminal illnesses are more likely to agree to having FP during resuscitation than those without terminal illnesses $(p=0.04)$. This could be due to the possibility that participants with terminal illnesses are more acutely aware of their desire to have their loved ones around in their final moments of life. Thus, FP during resuscitation would likely be more acceptable and useful for elderly patients and patients with terminal illnesses, as their family members may be more likely to be emotionally prepared to bid farewell. On the other hand, FP may not be a suitable option in situations involving paediatric patients, particularly in cases of sudden illnesses or traumatic events. Notwithstanding, the onus is on healthcare staff to change their perspective and allow family members the option of being present during resuscitation.

The present study is not without limitations. First, since this study involved a self-administered questionnaire, it is likely that persons who do not favour FP during resuscitation would be less willing to participate. Second, as this was a cross-sectional study, the opinions of our participants could change over time and ideas that may have seemed unfavourable at one particular point of time may become favourable at another time. For example, a participant who develops a chronic terminal illness at a later time in life may become more favourable toward the idea of FP during resuscitation. Finally, the sample size of the present study is small and may not truly represent the Malaysian population as a whole.

In conclusion, we found that the proportion of members of the Malaysian public who agreed that FP during resuscitation should be allowed is higher than that of Malaysian healthcare 
staff. This could be due to differences in concerns regarding the resuscitation process between members of the public and healthcare staff. Further studies should be conducted to investigate this postulate.

\section{REFERENCES}

1. Baumhover N, Hughes L. Spirituality and support for family presence during invasive procedures and resuscitations in adults. Am J Crit Care 2009; 18:357-66.

2. Mazer MA, Cox LA, Capon JA. The public's attitude and perception concerning witnessed cardiopulmonary resuscitation. Crit Care Med 2006; 34:2925-8.

3. MacLean SL, Guzzetta CE, White C, et al. Family presence during cardiopulmonary resuscitation and invasive procedures: practices of critical care and emergency nurses. Am J Crit Care 2003; 12:246-57.
4. Ersoy G, Yanturali S, Suner S, et al. Turkish patient relatives' attitudes towards family-witnessed resuscitation and affecting sociodemographic factors. Eur J Emerg Med 2009; 16:188-93.

5. Meyers TA, Eichhorn DJ, Guzzetta CE, et al. Family presence during invasive procedures and resuscitation. Am J Nurs 2000; 100:32-42.

6. Sheng CK, Lim CK, Rashidi A. A multi-center study on the attitudes of Malaysian emergency health care staff towards allowing family presence during resuscitation of adult patients. Int J Emerg Med 2010; 3:287-91.

7. Emergency Nurses Association (ENA). Position statement - Family presence during invasive procedures and resuscitation in the emergency department [online]. Available at: http://www.ena.org/SiteCollectionDocuments/ Position\%20Statements/FamilyPresence.pdf. Accessed June 2, 2013.

8. Ong ME, Chung WL, Mei JS. Comparing attitudes of the public and medical staff towards witnessed resuscitation in an Asian population. Resuscitation 2007; 73:103-8.

9. Timmermans S. High touch in high tech: the presence of relatives and friends during resuscitative efforts. Sch Inq Nurs Pract 1997; 11:153-68. 